MINI-SYMPOSIUM

\title{
Sirolimus eluting stent implantation for patients with multivessel disease: rationale for the arterial revascularisation therapies study part II (ARTS II)
}

\author{
P W Serruys, P A Lemos, B A van Hout, on behalf of the ARTS II Steering Committee and \\ Investigators
}

l: -stent restenosis and the need of repeat revascularisation remain the major limitations of coronary angioplasty for patients with multivessel disease. ${ }^{12}$ Utilisation of drug eluting stents in this context is expected to have a major impact on the effectiveness of percutaneous treatment. Sirolimus eluting stent implantation has recently been shown to significantly reduce coronary restenosis, with zero angiographic restenosis ${ }^{3}{ }^{4}$ and persistent long term neointimal inhibition. ${ }^{4}$ Moreover, sirolimus eluting stents were proven to be as safe as bare metal stents at 30 days in a group of patients with acute coronary syndromes and a high frequency of multivessel disease. ${ }^{5}$ However, currently the effect of this device on long term outcomes of patients with multivessel disease is largely unknown.

\section{EARLY CLINICAL EXPERIENCE: THE RESEARCH STUDY}

The sirolimus eluting stent received Conformité Européenne mark approval in April 2002, since when it has been available for routine use in Europe. From 16 April 2002, it has been the policy of our institution to utilise sirolimus eluting stents as the device of choice for all percutaneous coronary interventions, as part of the RESEARCH (rapamycin-eluting stent evaluated at Rotterdam Cardiology Hospital) study, which is a single centre registry conducted with the main purpose of evaluating the efficacy of sirolimus eluting stent implantation in the "real world".

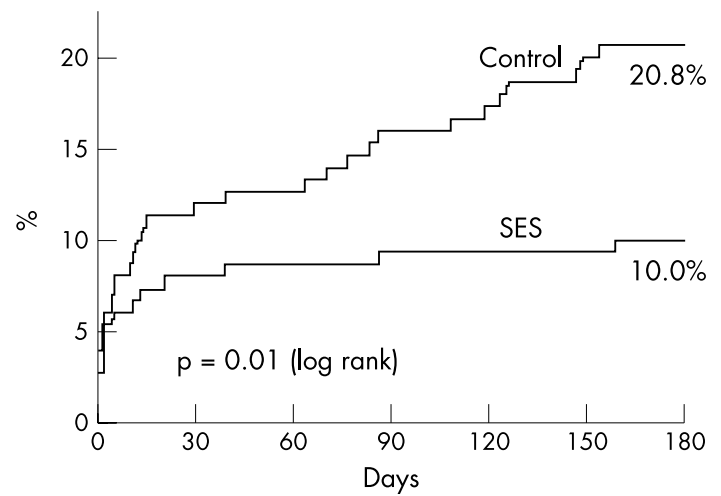

Patients at risk

Control $\quad 150$

SES $\quad 150$

126

134

Figure 1 Cumulative incidence of major adverse cardiac events (death, myocardial infarction, or repeat revascularisation) at six months for patients with multivessel disease treated with sirolimus eluting stents (SES) or bare metal stents (control). The incidence of repeat revascularisation during follow up is shown below.
In a preliminary analysis, the first 150 patients with multivessel disease included in the RESEARCH study were compared to a control group formed by 150 consecutive patients treated with bare stents in the period immediately before. Both groups were similar at baseline regarding the frequency of previous bypass surgery $(19 \% \vee 18 \%$, respectively; $\mathrm{p}=1.0)$, three vessel disease $(32 \% \vee 35 \%$, respectively; $\mathrm{p}=0.6)$, and diabetes $(27 \% \vee 22 \%$, respectively; $\mathrm{p}=0.4)$. At six months follow up, the cumulative incidence of major adverse cardiac events (death, myocardial infarction, or repeat revascularisation) was significantly lower among those treated with sirolimus eluting stents $(10.0 \% \mathrm{v}$ $20.7 \%$ in controls; $p=0.01$ ), mainly due to a pronounced reduction in the need for further revascularisation $(3.3 \% \mathrm{v}$ $12.7 \% ; p=0.003$ ) (fig 1). Although promising, however, these preliminary findings must be interpreted with caution until further confirmation with larger number of patients and more prolonged follow up is available.

\section{SIROLIMUS ELUTING STENT OR SURGERY FOR PATIENTS WITH MULTIVESSEL DISEASE? THE ARTS II STUDY \\ The premise and the philosophy of ARTS II}

Against the background of the impressive results of sirolimus eluting stent implantation in patients with single vessel disease and the promising initial findings of the RESEARCH study, the next ideal step should be a new randomised trial comparing treatment with multivessel stenting with the sirolimus eluting stent with the most advanced and up to date surgical treatment. However, before designing this new randomised trial it seems wise to improve the capabilities in treating complex multivessel disease (for example, main stem, total chronic occlusion, bifurcation, long lesion, small vessel). On the other hand, time is also needed in order to implement and vulgarise new surgical techniques that have been recently introduced and fully mastered in only certain centres of excellence. Therefore the ARTS II (arterial revascularisation therapies study part II) trial must be perceived as an intermediate phase towards the next full fledge second randomised ARTS trial. ${ }^{\circ}$

ARTS II is as a multicentre, non-randomised, open label, stratified trial designed to evaluate sirolimus eluting stent implantation in patients with multivessel disease using the surgical group of ARTS I as an historical control. To be comparable to the results of the ARTS I study, patients included in the ARTS II trial must present similar baseline

Abbreviations: ARTS, arterial revascularisation therapies study; CABG, coronary artery bypass graft surgery; CCS, Canadian Cardiovascular Society; MACCE, major cardiac and cerebrovascular events; RESEARCH, rapamycin-eluting stent evaluated at Rotterdam Cardiology Hospital 
characteristics. In order to ensure recruitment of a population very similar to the one of ARTS I, methodological techniques as "pseudo" randomisation or stratification will be applied.

\section{Objectives}

The main goal of the ARTS II trial is to assess a noninferiority of sirolimus eluting stent implantation for patients with multivessel disease with respect to the previous result of the surgical arm of the ARTS I trial.

The primary objective of ARTS II is to compare the effectiveness of coronary stent implantation using the sirolimus eluting Cypher (Cordis Johnson \& Johnson, Warren, New Jersey, USA) balloon expandable stent with that of surgery as observed in ARTS I. Effectiveness is measured in terms of major cardiac and cerebrovascular events (MACCE)-free survival at one year:

- death

- cerebrovascular event

- non-fatal myocardial infarction

- repeat revascularisation (either percutaneous or surgical).

The secondary objectives of this study are to compare the two groups with respect to:

- MACCE at 30 days, six months, and three and five years

- total cost at 30 days and cost, cost effectiveness, and quality of life at six months, and one, three, and five years

- combined end point death, myocardial infarction and stroke, and the itemised outcomes death, myocardial infarction, revascularisation procedure, stroke

- resource use at 30 days and one year.

\section{End point definitions \\ Death}

In case of death its exact cause will be recorded whenever possible on the basis of a necropsy. All deaths are considered cardiac unless there is documentation to the contrary. Secondary analysis will look separately at cardiac and noncardiac death.

\section{Cerebrovascular event}

Cerebrovascular events will be divided into three main categories: stroke, transient ischaemic attacks, and reversible ischaemic neurologic deficits.

\section{Myocardial infarction}

All myocardial infarctions will be counted as events, whether they occurred spontaneously or in association with angioplasty or coronary artery bypass graft surgery procedures. A definite diagnosis of myocardial infarction is made:

- first seven days post-intervention when both following criteria are positive:

- new abnormal Q waves not present at baseline (Minnesota code)

- creatinine kinase $2 \times$ normal (routine collection at six and 12 hours post-procedure and at discharge.

- after seven days from any intervention when at least two of the following occur:

- chest discomfort of at least 20 minutes duration

- new abnormal Q waves not present before allocation (Minnesota code)

- creatinine kinase $>2 \times$ upper limit.
Revascularisation procedure

Every subsequent revascularisation procedure is recorded, either percutaneous or surgical.

\section{Cost effectiveness}

Direct medical cost-Resource use concerning the initial procedure, hospital admissions, and major diagnostic or therapeutic procedures after the initial procedure.

Quality of life-The patient's quality of life will be assessed by the EuroQol and the SF-36 questionnaire (at randomisation, 30 days, six months, one year, three years, and five years). Furthermore, at the same point in time, the patient's anginal status will be assessed by the Canadian Cardiovascular Society (CCS) classification and the Braunwald classification. Before the procedure, and at all planned follow up visits, patients will be asked to fill out a questionnaire concerning their work status.

\section{Study population and stratification ("'pseudo randomisation"')}

A total of 600 eligible patients will be selected from the daily practices of 45-50 centres across Europe with a minimum enrolment of 12 eligible subjects per centre. Inclusion and exclusion criteria are presented in table 1. Patients are stratified by clinical site in order to ensure inclusion of one third of three vessel disease versus two thirds of two vessel disease, as based on the patient population enrolled in ARTS I. An ongoing check on the success of matching with the historical control will be performed. If it appears that the population has less than 2.7 lesions, the recruitment scheme of "3-2-2" will be changed to "3-3-2".

\section{Stent procedure and antiplatelet regimen}

The sirolimus eluting Cypher balloon expandable stent is premounted and crimped on the Raptor stent delivery system 5SDS. Direct stenting or pre-dilatation is left to the investigator's discretion. In case of inadequate positioning (uncovered lesion), dissection or unmasking of a narrowing in the inflow or outflow area of the implanted stent, a second or third stent may be placed in series. Intravascular ultrasound or any other monitoring devices are allowed. A multi-staged procedure is allowed and should be declared either before the initial procedure or at the time the patient is leaving the interventional suite. Patients who do not have all diseased vessels dilated, but all targeted vessels are successfully dilated, will be classified as "incompletely revascularised by intent". Those who do not have all targeted vessels successfully dilated will be classified as "incompletely revascularised, but not by intent".

Clopidogrel (preferably) or ticlopidine for eight weeks ( $75 \mathrm{mg} /$ day or $2 \times 250 \mathrm{mg}$ daily, respectively) and lifelong aspirin are commended. Clopidogrel (preferably) or ticlopidine should be used for at least six months for patients with diabetes or chronic total occlusion. Administration of glycoprotein IIb/IIIa inhibitors will only be considered in case of high instability, which cannot be controlled by any other means.

\section{Statistical analysis}

Assumptions for comparative analysis

Due to the expected pronounced decrease in the need for repeat revascularisations, the incidence of MACCE after sirolimus eluting stent implantation is probably best estimated as the proportion of patients in ARTS with death/ cerebrovascular accident/myocardial infarction (that is, 9.1\% after pooling the data from both ARTS treatment arms). ARTS II will not consider surgery as a separate arm and therefore the uncertainty margins need to be defined in advance. The ARTS I trial suggests a point estimate of $87.8 \%$ 
Table 1 Principal inclusion and exclusion criteria of ARTS II

Inclusion criteria

- Patients with stable (CCS 1, 2, 3, or 4) or unstable (Braunwald class IB, IC, IIB, IIC, IIIB, IIIC) angina pectoris considered for coronary revascularisation (angioplasty or $\mathrm{CABG}$ ). Patients with atypical chest pain or asymptomatic are eligible provided they have documented myocardial ischaemia

- At least 2 lesions de novo (located in different native vessels and in different territories) potentially amenable to stent implantation

- Multivessel disease with at least one significant stenosis ( $>50 \%$ by visual estimation) in the left anterior descending artery and with treatment of the lesion in another major epicardial coronary artery

- Left ventricular ejection fraction should be at least $30 \%$

- One total occluded major epicardial vessel or side branch can be included and targeted as long as one other major vessel has a significant stenosis amenable for stenting, provided the age of occlusion is less than one month. Patients with total occluded vessels of unknown duration or existing longer than one month and a reference over $1.50 \mathrm{~mm}$ should not be included

- Stenosis amenable to stenting by a stent ranging from $2.5-3.5 \mathrm{~mm}$

- Written informed consent

Exclusion criteria

- Under the age of 18 years, older than 80 years

- Previous CABG, PCl, or CVA

- Pregnant women or women with potential childbearing

- Clinical follow up over a period of five years judged to be difficult or unlikely or concomitant non-cardiac disease likely to limit long term prognosis

- Overt congestive heart failure

- Myocardial infarction $<7$ days

- Left main stenosis of $\geqslant 50 \%$

- Intention to treat more than one totally occluded major epicardial vessel

- A side branch larger than $1.5 \mathrm{~mm}$ covered by the stent constitutes an exclusion criterion, unless the operator is willing and technically able to maintain access to this side branch with either further balloon angioplasty (1.5$2.5 \mathrm{~mm}$ ) or stent placement $(>2.5 \mathrm{~mm})$

- Acute or chronic renal impairment (serum creatinine $>150 \mathrm{mmol} / \mathrm{l}$ or creatinine clearance $<50 \mathrm{ml} / \mathrm{min}$ )

- Known allergies to the following: aspirin, clopidogrel, ticlopidine, heparin, stainless steel, contrast agent (that cannot be managed medically), or sirolimus

CABG, coronary artery bypass graft surgery; CCS, Canadian Cardiovascular Society; CVA, cerebrovascular accident; $\mathrm{PCl}$, percutaneous coronary intervention;

with a $95 \%$ confidence interval of $83.4 \%$ to $92.3 \%$. In order to reflect the additional uncertainties due to eventual (minimal) changes of surgery over the time since the recruitment of the ARTS trial and/or mismatches between the two trials with respect to patient characteristics, the variance will be inflated by $25 \%$.

\section{Sample size}

For the sample size calculations several approaches were taken into consideration, both concerning clinical and cost and cost effectiveness outcomes, using Bayesian and frequentist methodology. It was decided to take the comparative statistical methodology, using a mixture of Bayesian and frequentist methodology.

A sample size of 600 patients is chosen to guarantee a power of at least $90 \%$. The sample size justification is based on the comparison of the ARTS I stented patients and the ARTS I surgery patients of the primary end point (one year MACCE). The null hypothesis is that the one year MACCEfree survival rate is at least as good as surgery minus 3.5\%, being $84.3 \%$. In the stented group an expected MACCE-free survival rate of $90.9 \%$ is used. This will be tested using a Bayesian approach. ${ }^{7}$ A one sided $t$ test will be performed with a type I error rate of 0.05 . Furthermore, an indication of the precision of the outcome of the ARTS II trial as a one arm registry is given as the width of the $95 \%$ two sided confidence intervals, being $4.8 \%$ (the $95 \%$ confidence interval around $90.9 \%$ is $88.3 \%$ to $93.1 \%$ ).

\section{Descriptive statistical methodology}

Safety data of the 30 days, six months, one, three, and five years will be analysed; however, the formal Bayesian analysis of the primary objective will only be performed at one year. A final analysis on the safety data will be performed at five years.
Effects

Count variables will be given as group rates and their matching 95\% confidence interval. Time-to-event variable will be presented as Kaplan-Meier curves. Continuous variables will be given as group means, and their matching $95 \%$ confidence intervals, and as the median, and the 10th and 90 th centiles.

\section{Costs and cost effectiveness}

Distinction is made between the direct medical costs and the total costs including the indirect medical costs. Emphasis is placed on the direct medical costs that are calculated as the product of the vector of resource used with a vector of unit costs. The indirect non-medical costs are addressed in terms of days of absence from work. In terms of effects, distinction is made between event-free survival and quality adjusted life years gained (estimated using two indices). As a result, six estimates result of the cost effectiveness ratio and the uncertainty surrounding these six estimates will be addressed by the methodology as outlined by van Hout and colleagues. ${ }^{8}$

\section{Comparative statistical methodology}

The primary analysis will be based on the intention-to-treat principle.

For the primary end point (MACCE at one year), comparing the ARTS II results with the historical controls of the ARTS trial, a Bayesian analysis will be used. Random and fixed effects will be pre-defined

Analysis with respect to the cost effectiveness end points will be performed for the predefined subanalysis: two versus three vessel disease, age, and insulin dependent diabetes.

\section{CONCLUSION}

The ARTS II study, with enrolment started in early 2003 and rapidly proceeding, should provide evidence of the effects of 
percutaneous coronary intervention with sirolimus eluting stents for patients with multivessel disease, as compared to a historical control group composed of the surgical arm of the ARTS I trial. The ARTS II trial should thereby help to clarify the impact of the new strategy in this context. Nevertheless, it is clear that during this decade further assessment in respect of the values of these two modalities of revascularisation should be assessed in a major and maybe final randomised trial.

\section{Authors' affiliations \\ P W Serruys, P A Lemos, Erasmus Medical Center, Thoraxcenter, Rotterdam, The Netherlands}

B A van Hout, Universitair Medisch Centrum, Julius Centrum, Utrecht, The Nertherlands

Correspondence to: Professor Patrick W Serruys, Thoraxcenter, Bd-406, Dr. Molewaterplein 40, 3015-GD Rotterdam, Netherlands; p.w.j.c.serruys@erasmusmc.nl

\section{REFERENCES}

1 Serruys PW, Unger F, Sousa JE, et al. Comparison of coronary-artery bypass surgery and stenting for the treatment of multivessel disease. N Eng J Med 2001;344:1117-24.

2 Pocock SJ, Henderson RA, Rickards AF, et al. Meta-analysis of randomised trials comparing coronary angioplasty with bypass surgery. Lancet 1995;346:1184-9.

3 Morice MC, Serruys PW, Sousa JE, et al. A randomized comparison of a sirolimus-eluting stent with a standard stent for coronary revascularization. N Engl J Med 2002;346:1773-80.

4 Degertekin M, Serruys PW, Foley DP, et al. Persistent inhibition of neointimal hyperplasia after sirolimus-eluting stent implantation: long-term (up to 2 years) clinical, angiographic, and intravascular ultrasound follow-up. Circulation 2002; 106:1610-3.

5 Lemos PA, Lee $\mathrm{CH}$, Degertekin $M$, et al. Early outcome after sirolimus-eluting stent implantation in patients with acute coronary syndromes-insights from the rapamycin-eluting stent evaluated at Rotterdam Cardiology Hospital (RESEARCH) registry. J Am Coll Cardiol 2003;41:2093-9.

6 Serruys PW. ARTS I-the rapamycin eluting stent; ARTS II-the rosy prophecy. Eur Heart J 2002; 23:757-9.

7 O'Hagan A, Stevens JW. A framework for cost-effectiveness analysis from clinical trial data. Health Econ $2001 ; 10: 303-15$.

8 van Hout BA, Al MJ, Gordon GS, et al. Costs, effects and C/E-ratios alongside a clinical trial. Health Econ 1994;3:309-19.

\section{IMAGES IN CARDIOLOGY}

\section{Aortic dissection with compression of the ostium of the right coronary artery: visualisation by 16 row} multislice CT

A

73 year old man with known coronary artery disease and acute coronary syndrome was referred for coronary angiography. ST element elevation occurred during contrast injection into the right coronary artery (RCA). Furthermore, contrast staining was detected close to the RCA ostium (below, left panel). Transoesophageal echocardiography (TOE) showed a focal dissection of the ascending aorta (below, middle panel). The patient was immediately referred for computed tomographic (CT) coronary angiography. The CT examination was performed using a 16 row multislice CT (Somatom Sensation 16, Siemens, Forchheim, Germany). Iodinated contrast (Xenetix 300, Guerbert GmbH, Sulzbach, Germany), $100 \mathrm{ml}$, was continuously injected into

an antecubital vein. The CT scan showed an intramural contrast depot and a focal dissection of the ascending aorta compressing the ostium of the right coronary artery (below, right panel). A coronary bypass operation was performed immediately. Two weeks later a follow up CT examination was performed which showed a patent venous bypass graft to the proximal RCA.

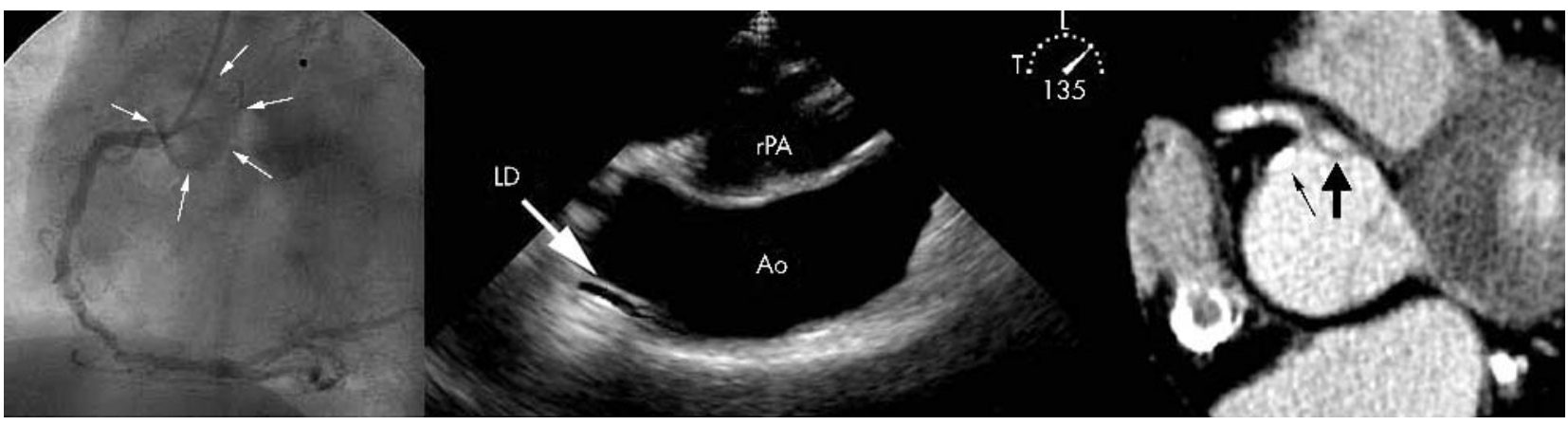

Left: Catheterisation image shows contrast staining around the RCA ostium (arrows). Middle: TOE shows a focal dissection (LD) of the ascending aorta (Ao) (rPA, right pulmonary artery). Right: Axial CT image demonstrating the dissection flap (thick arrow) of the aorta coursing to the RCA ostium and the proximal RCA. Intramural contrast depot at the RCA ostium (thin arrow). 